\title{
ESCENARIOS Y ESTRATEGIAS PARA EL APRENDIZAJE TRANSVERSAL
}

\author{
SCENARIOS AND STRATEGIES FOR TRANSVERSAL LEARNING
}

https://doi.org/10.15304/ie.30.7011

\author{
Carlos Rosales López \\ Universidade de Santiago de Compostela \\ carlos.rosales@usc.es
}

\section{RESUMEN}

Los objetivos de este trabajo se proyectan en la identificación de importantes escenarios y estrategias para el desarrollo de aprendizajes transversales, con especial hincapié en la educación para la salud. Se ha utilizado una metodología basada en el análisis de documentos correspondientes a organizaciones internacionales como la UNESCO y a la Administración educativa (legislación).

Se han analizado tres destacados contextos o escenarios: la administración educativa, el centro escolar y la ciudad educadora. Y se destacan como estrategias relevantes la adaptación de la enseñanza a los problemas de las personas y de la comunidad y el establecimiento de vínculos de colaboración entre instituciones y profesionales. En el ámbito de la metodología se destaca el valor interdisciplinar de los Proyectos de las Escuelas Asociadas de la UNESCO.

De manera específica, en el trabajo se analiza la incidencia de la pandemia del coronavirus en el funcionamiento de los colegios, que se han visto obligados a cerrar, a impulsar metodologías alternativas y ahora enfrentan el delicado proceso de su reapertura. La enorme transcendencia de esta enfermedad hace necesario replantear el fortalecimiento de la educación para la salud en todos los escenarios, para el desarrollo de aprendizajes sobre la prevención y la promoción de estilos de vida saludables.

Palabras clave: Educación para la salud; escuela saludable; ciudad saludable; proyectos interdisciplinares.

\begin{abstract}
The objectives of this work are projected in the identification of important scenarios and strategies for the development of transversal learning, with special emphasis on health education. A methodology based on the analysis of documents corresponding to international organizations such as UNESCO and the Educational Administration (legislation) has been used.

Three prominent contexts or settings have been analyzed: the educational administration, the school center and the educating city. And the adaptation of teaching to the problems of people and the community and the establishment of collaborative links between institutions and professionals
\end{abstract}

Recibido: 15/VII/2020. Aceptado: IX/2020 
stand out as relevant strategies. In the field of methodology, the interdisciplinary value of the UNESCO Associated Schools Projects is highlighted.

Specifically, the work analyzes the incidence of the coronavirus pandemic on the functioning of schools, which have been forced to close, promote alternative methodologies and now face the delicate process of reopening. The enormous importance of this disease makes it necessary to rethink the strengthening of health education in all settings, for the development of learning about prevention and the promotion of healthy lifestyles.

Key words: Health education; healthy school; healthy city; interdisciplinary projects.

\section{INTRODUCCIÓN}

En su origen, en nuestro país (LOGSE, 1990) los temas transversales constituyen contenidos de gran relevancia social y educativa como la convivencia, el medio ambiente, la salud o la igualdad que difícilmente pueden desarrollarse en el ámbito de las disciplinas convencionales o incluso en materias específicas creadas en torno a cada uno de ellos (Rosales, 2015). Se considera que es toda la escuela la que debe volcarse en el tratamiento educativo de estas cuestiones no solo por su significado social, sino también por la gran influencia que tienen en el desarrollo personal de cada uno de sus alumnos/as. Se hace necesaria una modificación cualitativa en la organización de las actividades y en la metodología de enseñanza. Y ello implica la adaptación de los proyectos educativos, así como de los diversos planes que los integran.

En el plan curricular, supuso un importante estímulo al desarrollo de los temas transversales la introducción con la LOE (2006) de las competencias básicas, que habían sido aprobadas por el Consejo de Europa como:

Aquellas que todas las personas precisan para su realización y desarrollo personal así como para la ciudadanía activa, la inclusión social y el empleo.

En su adaptación a la etapa de educación primaria, la Orden ECI/2211/2007 (MEC, 2007) las estructura en ocho tipos:

1. Competencia en comunicación lingüística. 2. Competencia matemática. 3. Competencia en conocimiento e interacción con el mundo físico. 4. Tratamiento de la información y competencia digital. 5. Competencia social y ciudadana. 6. Competencia cultural y artística. 7. Competencia para aprender a aprender. 8. Autonomía e iniciativa personal.

Con posterioridad también la LOMCE (2013) incluye en los planes curriculares las competencias básicas, si bien estructuradas en siete categorías:

1. Competencia lingüística. 2. Competencia matemática y competencias básicas en ciencia y tecnología. 3. Competencia digital. 4. Aprender a aprender. 5. Competencias sociales y cívicas. 6. Sentido de iniciativa y espíritu emprendedor. 7. Conciencia y expresiones culturales.

La toma en consideración de las competencia básicas como eje de las tareas curriculares debería imprimir al aprendizaje un carácter globalizador (actitudes, habilidades y conocimien- 
tos), una proyección en la realidad (capacidad de intervención) y un importante carácter interdisciplinar o transversal, ya que el desarrollo de estas competencias rebasa los límites específicos de cada asignatura, recabando la colaboración de todas o varias. Este carácter transversal se pone claramente de relieve en ámbitos como los lenguajes verbal y digital y las competencias sociales. Por extensión, en todas ellas.

La nueva ley de educación (MEC, 2020) confiere también un papel relevante a los aprendizajes transversales. Ya en el Proyecto aprobado en Consejo de Ministros en 15/02/2020 se proponen como pilares de la educación:

El cumplimiento efectivo de los derechos del niño según la Convención sobre los Derechos del Niño de Naciones Unidas (1989); el desarrollo de la educación en igualdad de género en todas las etapas educativas con especial referencia a la coeducación, la prevención de la violencia de género y el respeto a la diversidad afectivo-sexual; el desarrollo de un enfoque transversal para elevar los resultados de los estudiantes, la mejora de los centros educativos y la personalización del aprendizaje; la atención al desarrollo sostenible y la ciudadanía mundial de acuerdo con la Agenda 2030 y la toma en consideración del cambio digital en la comunidad y su repercusión educativa.

Así pues, ya en la declaración de principios se hace hincapié en la importancia de la transversalidad, en la globalidad del proceso educativo y de manera específica se incide en los temas de la igualdad, los derechos humanos, el desarrollo sostenible. La nueva ley modifica los artículos 18 y 19 de la LOE sobre los contenidos de enseñanza en la etapa de Educación Primaria. Pues bien, en el punto 10.3 se crea la asignatura de Educación en valores cívicos y éticos en tercer ciclo. En esta asignatura se contemplan contenidos relativos a los derechos humanos y de la infancia, la educación para el desarrollo sostenible y la ciudadanía mundial, la igualdad entre hombres y mujeres y el respeto a la diversidad. Por otra parte, en el apartado 11.3. se considera que:

Sin perjuicio de su tratamiento específico en algunas de las áreas de la etapa, la comprensión lectora, la expresión oral y escrita, la comunicación audiovisual, las tecnologías de la información y la comunicación, el fomento de la creatividad y del espíritu científico, la educación para la salud, incluida la sexual y la educación emocional y en valores, se trabajarán en todas las áreas.

En los apartados 16 y 17 de su artículo único, la ley en la organización de los contenidos de aprendizaje de la educación secundaria obligatoria introduce en alguno de los cursos la asignatura de Educación en valores cívicos y éticos y dispone también el tratamiento transversal, en todas las áreas de una serie de contenidos como los indicados en Educación Primaria.

Es muy probable que en línea con Europa y con lo dispuesto en la LOE (2006), también en los currículos de estas etapas aparezcan con un destacado protagonismo las competencias básicas. No obstante, a partir de la explicación de los pilares de la ley y de la organización prevista de los contenidos para estas etapas, es evidente que a los aprendizajes transversales se les confiere ya una relevante importancia. Por ello resulta evidente que en el centro escolar el desarrollo de estos temas va a implicar cambios significativos no solo en sus planes curriculares, sino también en los de orientación, de convivencia, de atención a la diversidad, lector..., que han de presentar una necesaria flexibilidad en cuanto a la utilización de estrategias y recursos 
alternativos, dedicación temporal y formas de colaboración. En el plan curricular será preciso introducir objetivos, contenidos y competencias vinculados con estos temas y organizarlos de forma secuencial e interdisciplinar. En el plan de orientación y acción tutorial se puede potenciar un asesoramiento al profesorado para el tratamiento de estos temas en las aulas y la atención personalizada a los alumnos, así como facilitar la colaboración del centro educativo con familia e instituciones de la comunidad. El plan de convivencia en los centros puede contribuir notablemente en la medida en que fomente el diálogo y la mediación así como la prevención, más allá de la necesaria normatividad. En el plan de atención a la diversidad los temas transversales se deberán adaptar a las características de los alumnos, considerando desde una perspectiva multicultural el distinto significado de temas como la igualdad o la convivencia.

En este proceso de cambio van a desempeñar un importante papel todos los profesionales implicados, como los directores/as de centros y muy especialmente el profesorado, que deberá ser capaz de rebasar el estrecho margen de sus disciplinas y responsabilidades individuales para embarcarse en el tratamiento educativo de cuestiones sociales novedosas y actuales, y deberá hacerlo en equipo, colaborando estrechamente con otros especialistas y padres.

\section{ESTRATEGIAS ADMINISTRATIVAS}

La Administración educativa a nivel central y autonómico, siguiendo orientaciones de carácter internacional, especialmente de la ONU y la UNESCO, se ha hecho eco de la necesidad de estimular la formación de las personas en temas tan importantes como la convivencia, la salud o la lectura comprensiva. En este sentido, el Ministerio de Educación ha puesto en marcha en 2017 los Planes Estratégicos de Convivencia Escolar y de Hábitos Saludables, así como el II Plan de Promoción de la Lectura. Estas estrategias y planes del Ministerio han encontrado continuidad en las Comunidades Autónomas, que elaboran sus propios planes adaptados

De manera específica, en el ámbito de la convivencia se propusieron siete importantes núcleos de actividades vinculadas con la educación inclusiva, la participación en la comunidad educativa, el aprendizaje y la convivencia, la educación en los sentimientos y en la amistad, la socialización preventiva de la violencia desde la primera infancia y la atención y cuidado del uso de la tecnología de la información y la comunicación.

En principio se preveían actuaciones relacionadas con la celebración anual de un congreso estatal de convivencia escolar, la creación en los colegios de una figura responsable de la convivencia y la puesta en funcionamiento de una App de ayuda a víctimas de acoso escolar. Se elaboraron guías para la prevención y apoyo a víctimas de acoso y de ciberacoso en el contexto escolar.

En el Plan Estratégico de Salud Escolar y Estilos de Vida Saludables se contemplan como líneas importantes de actuación:

Crear marcos de colaboración institucional entre administraciones y agentes implicados en la promoción de estilos de vida saludables, diseñar estrategias para la promoción de la salud en el ámbito educativo, crear y desarrollar la Red Estatal de Escuelas Promotoras de Salud, fomentar 
la formación del profesorado, investigar y evaluar los avances en formación de estilos de vida saludables (MECD, 2017a).

De manera específica se contempla la creación de un sello distintivo de centros escolares con buenas prácticas en educación para la salud. Se contempla también la creación de una asignatura sobre promoción y educación para la salud en los planes de formación docente y la creación de talleres en los que padres y alumnos progresen en la mejora de hábitos saludables.

En estos mismos años, el MEC puso en marcha el II Plan para el Fomento de la Lectura (2017-2020), que se apoya en la Ley 10/2007 de la lectura, el libro y las bibliotecas, así como en la Ley Orgánica de Educación (LOE, 2006), en la que se considera la lectura como un tema transversal al mismo nivel que los relativos a la convivencia, el medio ambiente, la salud o la igualdad. En el II Plan se asume que la extensión de la capacidad lectora es una responsabilidad globalmente social y por lo tanto, se considera que son muchas las instituciones, no solo la escolar, las que deben implicarse responsablemente. De hecho, en el Plan se incluyen seis líneas de intervención relativas a:

1. La promoción de hábitos lectores. 2. El impulso de la lectura en el ámbito educativo. 3. El refuerzo del papel de las bibliotecas en el acceso a la lectura. 4. El fortalecimiento del sector del libro. 5. El fomento del respeto a la propiedad intelectual. 6. La mejora de las herramientas de análisis. (MECD, 2017b)

Las estrategias y planes impulsados por la administración central, en función de orientaciones internacionales han tenido continuidad en otros más específicos elaborados en cada Comunidad Autónoma, de tal modo que en la actualidad, los centros escolares al tratar de desarrollar los denominados temas transversales, cuentan con un marco referencial y de apoyo múltiple, constituido por la comunidad próxima o municipal, por las administraciones autonómica y estatal y por organizaciones internacionales como la ONU, la UNESCO, la OMS y otras.

\section{ESCUELAS SALUDABLES}

El proceso cualitativo de cambio para el tratamiento educativo de los contenidos transversales podría estudiarse específicamente para cada uno de los temas en la actualidad relevantes. Podríamos pensar en una escuela que priorice la educación en la convivencia y en el desarrollo sostenible o en una escuela que ponga especial interés en la educación para la igualdad y la inclusión. En la actualidad y en función de la problemática originada por la pandemia del coronavirus, resulta evidente la necesidad de priorizar la educación para la salud, en cuyo caso la escuela probablemente se aproximaría al modelo de las Escuelas saludables.

Tomando como punto de partida la consideración de la salud como un relevante tema de interés social y personal, fundamental para la conservación de la vida y para la realización satisfactoria de cualquier actividad. Tomando en consideración que en determinadas etapas o momentos de la historia, la salud individual y social se ve amenazada por enfermedades tan 
peligrosas como la actual pandemia por coronavirus, todas las instituciones sociales y entre ellas la escuela deben contribuir al mantenimiento de la salud y el alejamiento de los riesgos de enfermedad. En este caso la educación para la salud, se entiende como prevención y promoción de hábitos saludables, como capacitación de las personas para que puedan controlar los factores que afectan a su salud y la de su comunidad, y en el caso de la pandemia actual, como información y desarrollo de actitudes y habilidades fundamentalmente preventivas.

Entre los antecedentes para la creación de escuelas saludables se suele citar la Carta de Ottawa, de la OMS (1986). Con el objetivo de Salud para todos en el año 2000, se establece claramente en ella la necesidad de promover de la salud en los entornos de vida de las personas. En este sentido se dice en la Carta:

Es esencial proporcionar los medios para que a lo largo de su vida la población se prepare para las diferentes etapas de la misma y afronte las enfermedades y lesiones crónicas. Esto se ha de hacer posible a través de las escuelas, los hogares, los lugares de trabajo y el ámbito comunitario en el sentido de que exista una participación activa por parte de las organizaciones profesionales, comerciales, y benéficas, orientada tanto hacia el exterior como hacia el interior de las instituciones mismas.

Y en otro apartado se dice:

La salud se crea y se vive en el marco de la vida cotidiana, en los centros de enseñanza, de trabajo y de recreo.

Organismos como la propia OMS, la UNESCO, el UNICEF, el Consejo de Europa y la Comisión Europea se han manifestado a favor de la promoción de la salud en el ámbito educativo. Y en 1991 por iniciativa de la Oficina Regional de la OMS para Europa, en colaboración con la Comisión Europea y el Consejo de Europa, se crea la Red Europea de Escuelas Promotoras de Salud (REEPS). Actualmente se denomina SHE (Escuelas para la Salud en Europa). Su coordinación se encuentra en Holanda. Cada uno de los 45 Estados que forman parte de la red tiene un coordinador nombrado por los Ministerios de Educación y Sanidad. En este contexto y con objeto de potenciar a nivel Estatal el desarrollo de las escuelas para la salud, el Ministerio de Educación ha impulsado la creación de la Red Estatal de Escuelas Saludables, calificando así a los centros educativos que se esfuerzan por crear un entorno físico y psicosocial promotor de bienestar y estilos de vida saludables en todos sus componentes y en estrecha colaboración con otros centros, así como con las familias y la comunidad. Para ello se considera necesario desarrollar el sentido de la participación responsable de todos los miembros del centro, así como la introducción de contenidos y prácticas sobre la educación para la salud en el proyecto educativo y en todos los planes y programaciones. Se hace uso de una amplia variedad de recursos propios y externos.

Desde la perspectiva de un tratamiento transversal de la educación para la salud, los centros escolares deberían contar con un marco legal más amplio y flexible que el existente en la actualidad. Así, en la etapa de educación primaria tan solo un objetivo (k), se proyecta en la salud y lo hace de forma limitada, centrándose en la dimensión física, sin mencionar compo- 
nentes mentales y sociales de la misma. Por otra parte, las competencias básicas tampoco mencionan de forma explícita la educación para la salud. Sería muy conveniente una explicación de las formas en que dichas competencias comprenden también la educación en cuestiones de salud. En la consideración que se hace de elementos transversales para esta etapa, en el decreto de currículo no se menciona la educación para la salud. Y en la organización de contenidos, objetivos y competencias para cada materia, tan solo en las de ciencias naturales y educación física aparecen temas vinculados con la salud y referencias al objetivo k) de la etapa. Todo ello desde una perspectiva predominantemente física (ejercicio, alimentación, higiene).

\section{CIUDADES SALUDABLES}

La actuación de cada escuela se potencia en la medida en que establece vínculos de colaboración con otras escuelas y de manera amplia, con instituciones diversas de carácter social, cultural, artístico, natural... De hecho, en la actualidad existe en los municipios una creciente sensibilidad hacia la facilitación y desarrollo de proyectos de carácter educativo, que se manifiesta de muy diversas maneras. En general se potencian la información y la participación de los ciudadanos en temas de la comunidad, se desarrollan formas de intervención educativa formal y no formal extendidas a todas las generaciones y con especial atención a gentes que se encuentran en riesgo de exclusión social. Se fomenta un ocio constructivo y la orientación profesional con medidas facilitadoras de empleo. Se establecen vínculos de colaboración con centros escolares subvencionando algunas de sus actividades, permitiendo el acceso a instalaciones deportivas, explicando el funcionamiento de los servicios municipales, fomentando una proyección pedagógico-didáctica en el uso de sus museos y otras instituciones, etc.

Esta proyección educativa de los municipios se pone de relieve específicamente en documentos como la Carta de las Ciudades Educadoras, aprobada en el I Congreso Internacional de Ciudades Educadoras celebrado en Barcelona en 1990 y actualizada en sucesivos congresos como el de Bolonia en 1994 y el de Génova en 2004. Su fundamento se encuentra en la Declaración Universal de Derechos Humanos de 1948, la Convención sobre Derechos de la Infancia de 1989, la Declaración Mundial de Educación para Todos de 1990 y la Declaración Universal sobre Diversidad Cultural del 2001. A lo largo de los artículos de que se compone, se manifiesta una clara preocupación por la formación en valores de convivencia. Así, en su artículo veinte se dice:

La ciudad educadora deberá ofrecer a todos sus habitantes como objetivo crecientemente necesario para la comunidad, formación en valores y prácticas de ciudadanía democrática: el respeto, la tolerancia, la participación, la responsabilidad y el interés por lo público, por sus programas, sus bienes y sus servicios.

Las ciudades que manifiestan una especial preocupación y compromiso con la salud de sus ciudadanos, se consideran ciudades saludables. Su origen se sitúa en los años ochenta del pasado siglo en Pécs, Hungría, la Conferencia internacional sobre ciudades saludables. En ella los alcaldes y líderes políticos aprobaron una Declaración en la que reafirman sus objetivos originales: 
Somos conscientes de que hacer de la mejora de la salud y el bienestar de todos una prioridad para las ciudades es crucial para sentar las bases de un futuro de desarrollo sostenible y saludable. Reconocemos nuestra responsabilidad política de trabajar hacia la creación de sociedades que sean inclusivas, seguras, resilientes y sostenibles y que permitan a todas las personas llevar una vida más empoderada, saludable, feliz, segura y satisfactoria.

Existen redes nacionales de ciudades saludables en numerosos países. En 1988 nace la red española con la participación del Ministerio de Sanidad, la Federación de Municipios y Provincias y el Ayuntamiento de Barcelona. De ella forman parte más de treinta capitales de provincia y numerosas ciudades de más de cien mil habitantes. La pertenencia a la red aporta a las ciudades más información y apoyo en la realización de proyectos, en los que son fundamentales la cooperación intersectorial y la participación ciudadana. En las ciudades saludables se procura la satisfacción de las necesidades básicas de las personas como condición fundamental para su bienestar físico, mental y social. De manera específica se trata de promover el desarrollo de hábitos y estilos de vida saludables a través del estímulo a la actividad física, la alimentación sana, la reducción del consumo de sustancias aditivas, la prevención de enfermedades, el diagnóstico temprano y la rehabilitación. La intervención se proyecta en todas las edades y situaciones de las personas. Existe un especial interés en motivar e incrementar la participación de los ciudadanos en los programas de salud.

\section{ACTIVIDADES COMPLEMENTARIAS}

Una estrategia clásica de comunicación y colaboración entre la escuela y su entorno son las salidas o actividades complementarias. Esta última denominación es en realidad engañosa pues las considera como un simple apéndice de las actividades curriculares, cuando en realidad implican la realización de aprendizajes muy importantes, que se pueden convertir en punto de partida de numerosas tareas escolares. Así lo han considerado destacados educadores en todos los tiempos y de manera especial los componentes de la Escuela Nueva. También en la Institución Libre de Enseñanza (ILE) se las potencia de manera importante. En el gobierno de la II República incluso se introdujo una asignatura sobre las mismas en el plan de estudios de Maestro. Tras el frustrante periodo de la Dictadura, en el que estas actividades se vieron reducidas al plano de lo anecdótico, se recobra nuevamente su valoración con el empuje educativo de los Movimientos de Renovación Pedagógica (MRP) a partir de los años sesenta del siglo XX (Rogero, 2010). En la actualidad se contempla legalmente su realización en los Reglamentos Orgánicos de Centros de Educación Primaria y Secundaria.

Se constata la existencia de una amplia aceptación de estas actividades complementarias o salidas, tanto por profesores como por alumnos y padres, viéndose limitadas solamente por factores como el económico, la preparación y disponibilidad de tiempo y condiciones organizativas y la existencia de oportunidades de apoyo institucional extraescolar (García Gómez y Cabanillas, 2016).

Son muchas las posibilidades de realización de salidas escolares. Podríamos pensar en la duración de las mismas, en cuyo caso nos podemos referir a salidas de corta duración como 
las practicadas por Freinet (1973) con sus alumnos por las tardes para conocer características de distintos oficios en el pueblo donde se ubicaba la escuela, o las salidas que en la actualidad realizan colegios de centros urbanos para asistir a conciertos didácticos o a sesiones de cine en valores o para conocer instalaciones y servicios municipales. También se puede hacer referencia a salidas de larga duración (varios días), como excursiones de fin de curso o de etapa. Si tomamos como referencia el entorno, se pueden citar las salidas de toma de contacto con la naturaleza, desde la realización de itinerarios ecológicos a la realización de observaciones y experiencias de tipo natural en un bosque próximo al colegio, las llamadas aulas de la naturaleza (Austin, 2009). También se puede destacar aquí el interés de la visita a granjas-escuela.

En el análisis de las repercusiones pedagógicas, didácticas y organizativas de las salidas escolares se pone de relieve la existencia de considerables ventajas educativas. Los alumnos/ as realizan importantes aprendizajes directos a través del contacto, observación e interacción con la realidad y este aprendizaje es en parte por descubrimiento, pero en gran medida por descubrimiento guiado (Benejam, 2003), en función de la preparación de que habrá sido objeto por los profesores la salida escolar. Así, en un aula de la naturaleza pueden realizar registros meteorológicos, cálculos, representaciones, informes, dibujos o grabaciones. En un museo interactivo pueden realizar observaciones y experiencias muy próximas a la realidad. Las salidas se convierten las más de las veces en origen de una pluralidad de aprendizajes que presentan una doble dimensión: convencional y transversal.

Así, en la técnica Seguir la pista, utilizable en educación para el consumo en tercer ciclo de educación primaria, los alumnos/as eligen un producto de su entorno (por ejemplo, una conserva alimentaria) y realizan un seguimiento de la misma desde la identificación de las materias primas, su extracción y transporte, el proceso de fabricación, difusión en mercados y publicidad, características ecológicas de los residuos que origina su fabricación, sus envases, etc. Probablemente habrán tenido que realizar más de una salida del centro escolar: toma de contacto con el contexto del que se extraen las materias primas, visita a la fábrica en la que se realiza el producto, visita a un mercado en el que se distribuye y vende. Paralelamente habrán llevado a cabo interesantes experiencias de trabajo en equipo y resolución de problemas. Desde una perspectiva curricular convencional son muchas las materias que se verán implicadas en mayor o menor medida como las ciencias naturales (materias primas, procesos de elaboración), las ciencias sociales (observación, entrevistas, cuestionarios para el conocimiento de cuestiones laborales, formación para el trabajo, especialización), lenguaje (consulta en diversas fuentes, realización de informes), matemáticas (solución de problemas, cálculos), expresión artística (dibujos, representaciones), etc.

Desde una perspectiva organizativa, la realización de esta técnica implica el trabajo en equipo del profesorado, del que está más directamente vinculado con los contenidos que se trabajan. Los profesores han de ser capaces de sobrepasar los límites de tiempo, del aula y del colegio y de los contenidos de los que son directamente responsables para establecer objetivos y desarrollar actividades en colaboración con otros profesores y profesionales. 


\section{SUCESOS OCASIONALES}

Otra forma de tomar contacto con el entorno consiste en el tratamiento pedagógico/didáctico de acontecimientos inesperados que afectan fuertemente a la comunidad y al contexto natural, ante los cuales la escuela no puede ser indiferente. Se establece como un paréntesis en el desarrollo rutinario de la actividad escolar para dar cabida en las aulas a importantes cuestiones, generalmente de carácter transversal, vinculadas con la convivencia, el medio ambiente, la salud, la igualdad, etc. Paralelamente al trabajo en el tema ocasional, los alumnos/as tendrán la oportunidad de realizar avances en los contenidos convencionales.

Así, en Galicia, en el mes de noviembre de 2002, el buque petrolero Prestige sufrió una rotura de tanques a consecuencia de un golpe de mar a cincuenta kilómetros de Finisterre. El buque acabó hundiéndose y vertiendo más de setenta mil toneladas de un fuel especialmente tóxico. Grandes sectores de la costa gallega se vieron afectados en lo que ha constituido la mayor catástrofe ecológica de su historia. Playas, rocas, fondo marino resultaron contaminados, afectando de manera muy importante a las formas de trabajo y de vida de numerosas gentes. Muy pronto sin embargo, se produjo una respuesta social constructiva. Incluso antes de que las instituciones oficiales pusieran en marcha planes de recuperación, aparecieron cientos de voluntarios de todo el país y del extranjero que actuaron con gran eficacia desde un primer momento en la limpieza del agua y las costas.

Entre dichos voluntarios desempeñaron un destacado papel los estudiantes universitarios, especialmente de las tres universidades gallegas, que aplazaron por días sus estudios para realizar una importante labor social. En esas jornadas de trabajo pusieron en práctica importantes valores de ciudadanía y solidaridad, así como de recuperación ambiental. Las tareas, realizadas en equipo, constituyeron una singular oportunidad para la cooperación entre ellos y con las gentes de diversas localidades costeras. Alumnos más jóvenes y niños que no pudieron trabajar con los adultos, llevaron a cabo otras formas de actividad como manifestaciones de la mano de sus padres o formación de cadenas humanas. De puertas adentro, en los centros escolares se realizaron una enorme cantidad de trabajos. Los alumnos/as tuvieron la oportunidad de hablar y debatir sobre numerosas cuestiones, realizar informes, proyectar el tema central del Prestige en contenidos de carácter convencional, realizando así aprendizajes en el ámbito de la geografía, meteorología, historia, matemáticas, ecología, lengua, etc. (Rosales, 2005)

Los sucesos ocasionales, como las salidas, presentan un alto efecto motivador al poner en contacto al alumno con la realidad vital. Sus aprendizajes tienen un carácter muy funcional, dado que se realizan en el marco de proyectos y objetivos que afectan a su entorno natural y social. Otra característica importante de ambas actividades es el realizarse generalmente en equipo, fomentando la comunicación y colaboración con los compañeros/as y los profesores/ as. Se pone especialmente de relieve en las salidas de larga duración, en las que la convivencia, más allá del estricto horario lectivo hace que se lleguen a conocer desde una perspectiva ampliamente humana. 


\section{LA CRISIS DEL CORONAVIRUS Y EL APRENDIZAJE TRANSVERSAL}

La crisis originada por la pandemia del coronavirus está poniendo de relieve la naturaleza transversal de los aprendizajes sobre la salud, una transversalidad que no solo pone a prueba las posibilidades de los centros escolares como escuelas para la salud, sino también las formas de colaboración de la escuela con la familia y la comunidad. Resulta más necesaria que nunca la colaboración de maestros y especialistas con padres y madres. De hecho durante varios meses, las horas que el niño pasaba en la escuela, al cerrar esta se han trasladado al hogar y los padres han tenido que actuar como maestros, al tiempo que estos han sustituido la relación directa con sus alumnos por la comunicación a través de internet. La modificación de los hábitos de vida ha sido tan intensa y repentina que todo el aprendizaje y la actividad educativa parecen subordinarse al desarrollo de habilidades y actitudes de supervivencia. Adultos y niños hemos tenido que aprender de forma urgente conocimientos básicos de higiene, relación a distancia y utilización de mascarillas. Hemos modificado nuestras formas de trabajo y de ocio y desarrollado importantes rasgos de voluntariado, servicio y solidaridad. Hemos aprendido a valorar nuestro propio equilibrio emocional y social.

La vuelta a la normalidad, la vuelta a las aulas va a implicar importantes cambios en cuanto al uso de espacios, formas de relación, normas de higiene y protección, pero también y considero que esto es muy importante, en cuanto se refiere a la determinación de objetivos y contenidos, incidiendo en la influencia de las enfermedades epidémicas, en la importancia de la salud emocional, en el uso de nuevas tecnologías y programas de aprendizaje a distancia y en la colaboración del centro escolar con las familias y otras instituciones. La transversalidad curricular de la educación para la salud se pone tan claramente en evidencia que difícilmente ninguna asignatura podrá cerrarse ante la necesidad de colaborar interdisciplinarmente en este tipo de aprendizaje.

En su guía para la reapertura de los centros escolares tras su cierre por el coronavirus, la UNESCO (2020b) reflexiona sobre el perjuicio que el cierre de los mismos ha ocasionado en niños/as de todo el mundo, sobre todo en los países más empobrecidos. El cierre de escuelas ha afectado a más de mil trescientos millones de alumnos/as y ha implicado importantes riesgos. Al cerrar las escuelas se han visto privados de servicios de alimentación y salud que normalmente se prestan a través de ellas. El aprendizaje se ha perturbado considerablemente ya que las estrategias alternativas con uso de recursos en línea no siempre se han podido implementar a un nivel mínimo de eficacia. Además y sobre todo en países empobrecidos, se han incrementado los problemas de trabajo infantil, matrimonios prematuros, abandono escolar por alumnos de minorías que ya estaban precariamente escolarizados. La nueva situación, con pérdida de contacto de los niños/as con sus compañeros/as ha influido negativamente en su estabilidad psicosocial.

La UNESCO considera que ante la necesaria reapertura de los centros escolares, las administraciones han de tomar en consideración una serie de orientaciones o directivas que se proyectan en ámbitos como los siguientes: 
a) Desde una perspectiva política y de financiamiento se ve necesario que las administraciones promuevan una serie de normas claras en torno a la apertura y funcionamiento de los centros.

b) Considera que se deben fortalecer los sistemas educativos incrementando los niveles de atención a todos los sectores de población en condiciones de igualdad y equidad.

c) Se hace hincapié también en la necesidad de garantizar un trabajo seguro en los centros para todo el personal, estableciendo las adecuadas prácticas de higiene, espacios, protección...

d) De manera específica en relación con los alumnos/as se considera prioritario restablecer medidas vinculadas con su bienestar en los ámbitos de la alimentación, la salud y el equilibrio psicosocial.

e) Es preciso incidir especialmente en la recuperación de aprendizajes fundamentales y establecer para ello programas adaptados, en los que los temas vinculados con la salud deberán ocupar un lugar relevante.

Se trata de directrices en gran medida aplicables de forma general, si bien la UNESCO explica que deberán adaptarse a las condiciones locales. En este sentido nuestros centros educativos en la actualidad, al priorizar la salud de sus alumnos y profesores tienen que plantearse un uso diferenciado de espacios, una adecuada disponibilidad y uso de recursos de higiene, la utilización de adecuados protocolos de intervención tanto dentro como fuera de las aulas y de relación con familiares. Asimismo, desde una perspectiva organizativa, debemos pensar en una modificación de horarios, en la disminución del número de alumnos por aula, en la combinación de sesiones presenciales con trabajo individual. En el caso específico del trabajo en línea será preciso procurar la igualdad de oportunidades en cuanto a disponibilidad y uso de instrumentos. Debemos pensar también que las funciones orientadora y tutorial del centro y los profesores van a adquirir un especial protagonismo.

\section{PROYECTOS INTERDISCIPLINARES}

La institución más representativa a nivel internacional de la promoción de proyectos transversales interdisciplinares con proyección sociocultural y ambiental es la UNESCO. Surgida en 1945, una vez terminada la Segunda Guerra Mundial (IIGM), con antecedentes en la CAME (Conferencia de Ministros Aliados de Educación), que había tenido lugar en 1942 en pleno conflicto, la UNESCO se va a responsabilizar de la educación, la ciencia y la cultura en todo el mundo, con especial incidencia en el desarrollo de temas de convivencia, medio ambiente e igualdad. En estos momentos asume la coordinación de acciones en torno al desarrollo de los objetivos en educación de la Agenda de las Naciones Unidas para 2030. En la programación para el periodo 2014-2021 se compromete con objetivos como la formación de las personas como ciudadanos del mundo, creativos y responsables, la extensión del aprendizaje a lo largo de toda la vida y el apoyo a los estados miembros en el desarrollo de sistemas educativos de calidad. 
La UNESCO en el terreno educativo hace operativas sus aspiraciones a través fundamentalmente de las Escuelas Asociadas, constituidas en red mundial de escuelas para abordar retos mundiales, en estos momentos, más de once mil distribuidas en prácticamente todos los países. Esta Red ha adoptado de cara a los objetivos para el periodo 2014-2021 dos importantes estrategias relativas a considerar la educación para la ciudadanía y la educación para el desarrollo sostenible como prioritarias en el conjunto de su intervención educativa. En educación para la ciudadanía pretende promover la formación en niños y jóvenes de la capacidad para implicarse en la búsqueda de soluciones a problemas mundiales. Y considera que para ello es preciso estimular su aprendizaje en un triple sentido: cognitivo, socioemocional y conductual.

En la estrategia de educación para el desarrollo sostenible la Red de Escuelas subraya la importancia de fomentar en los alumnos conocimientos teóricos y prácticos para enfrentarse a los problemas de forma constructiva y creativa. Se considera que su aprendizaje se ha de proyectar en el triple ámbito de las actitudes, las habilidades y los conocimientos. En los contenidos de enseñanza deberán incluirse temas de carácter local y global y deberán desarrollarse a través de actividades participativas.

En relación con sus estrategias educativas de carácter general, la UNESCO desarrolla proyectos específicos con mayor o menor amplitud territorial y temporal. Algunos de ellos han alcanzado notable éxito y prestigio como Linguapax, que tiene su origen en 1987 y cuyo objetivo es el desarrollo de las lenguas y a través de ellas, de la paz entre los pueblos. Este proyecto dio lugar a una organización con sede en Barcelona y estatus consultivo ante la UNESCO, cuyos objetivos en parte son coincidentes con los objetivos en educación del programa 2030 de la UNESCO y se proyectan básicamente en la promoción, preservación y activación de la diversidad lingüística. (Linguapax, 2020).

Dentro del proyecto Linguapax un conjunto de escuelas asociadas de Euskera, Cataluña y Galicia, han elaborado un libro, Un barco de poemas, editado por la ikastola Artxandape. En él se recogen trabajos de profesores y alumnos pertenecientes a estas escuelas. Con esta obra se quiere realizar un homenaje y dar a conocer las diversas lenguas oficiales del Estado.

\section{Proyecto del Mar Báltico}

Con la participación de nueve países: Dinamarca, Estonia, Finlandia, Alemania, Letonia, Lituania, Polonia, Rusia y Suecia, y trescientas escuelas asociadas, es el proyecto internacional más antiguo en educación ambiental. A lo largo de los años dentro del proyecto se han realizado interesantes materiales y seminarios didácticos. Entre sus objetivos se destaca además del aprendizaje medio-ambiental, la educación intercultural. Son múltiples las actividades realizadas por los alumnos, tanto dentro como fuera de las aulas: características de las costas, calidad del agua, historia, etc. (Kakse, 2019).

\section{Proyecto Mediterráneo Occidental}

Se trata de un proyecto puesto en marcha en 1992 por la UNESCO, en el que participan los países del Mediterráneo Occidental: España, Francia, Grecia, Italia, Malta, Marruecos, Túnez, Andorra y Libia. Presenta una incidencia positiva en el conocimiento de las características 
medio ambientales y culturales de la zona y en el establecimiento de vínculos de comunicación, interacción y colaboración (UNESCO, 2020a).

En este proyecto se trabajan temas relativos a las características de las gentes del litoral, la biodiversidad, la desertización y el paisaje. Las escuelas participantes realizan materiales y establecen correspondencia entre ellas, así como se intercambian recursos y elaboran una revista en común.

\section{Proyecto: El patrimonio en manos de los jóvenes}

Tiene su origen en el curso 1994-95 como una colaboración de Escuelas Asociadas con el Patrimonio Mundial. Se propone sensibilizar a los jóvenes y darles a conocer la importancia de defender el patrimonio cultural y natural del mundo a partir de la realidad de su entorno, inscribiéndola en la global de la humanidad. De manera específica trata de promover el conocimiento de los lugares culturales y materiales de sus países y de otros. Se fomenta también el conocimiento de los oficios tradicionales para la conservación y restauración del patrimonio. En el desarrollo de este proyecto, como en otros, se utiliza una metodología interdisciplinar con actividades extraescolares y se elaboran interesantes materiales didácticos (Morillas, 2006).

\section{Proyecto: La ruta del esclavo. Romper el silencio}

Tiene su origen en 1994 con un triple objetivo: poner de relieve las causas y la naturaleza del comercio transatlántico de esclavos, estudiar sus consecuencias en África, América y Europa y fomentar una cultura de coexistencia pacífica entre los pueblos. En relación con este proyecto, desde 1998 la Red de Escuelas Asociadas desarrolla el proyecto titulado Romper el silencio, en el que se estudian formas de discriminación específicas como el racismo y el apartheid. Y en el año 2005 se pone en marcha la campaña Todos iguales en la diversidad, para movilizar las escuelas en torno a problemas como el racismo, la discriminación y la exclusión. Un grupo de escuelas asociadas ha trabajado en torno a este proyecto centrándose en el papel de la música como acompañante de los esclavos en su desgracia, analizando la influencia de la música sobre ellos y en los pueblos en los que vivieron, así como su presencia actual. (Tutor, 2006).

\section{CONCLUSIONES}

Los aprendizajes transversales han sido introducidos y promovidos en los niveles de educación obligatoria por las leyes educativas de las últimas décadas desde la LOGSE (1990) a la LOMLOE (2020). Y en consecuencia, se contemplan en los decretos de currículo actuales. Entre otros temas como el de la igualdad, la convivencia pacífica, el medio ambiente o los lenguajes, figura desde el primer momento el de la educación para la salud. La Administración educativa complementariamente a la legislación básica, han puesto en funcionamiento además estrategias específicas para el desarrollo de temas como la convivencia, la salud y la lectura, en el periodo comprendido entre 2017 y 2020 . Concretamente en la estrategia sobre salud se propone la creación de la Red Española de Escuelas Saludables. 
En el ámbito del centro escolar el desarrollo de los temas transversales y en concreto, de la educación para la salud implica importantes modificaciones cualitativas que se reflejarán en su proyecto educativo y en los planes de que se compone como el plan docente, el de convivencia, el de orientación y tutoría, el lector, el de atención a la diversidad, así como en las relaciones que mantiene con las familias y la comunidad. Una escuela que prioriza la educación para la salud en sus características organizativas y metodológicas se aproxima al modelo de Escuelas Saludables. Y su actuación se puede potenciar considerablemente en la medida en que se encuentre ubicada en una Ciudad Saludable, esto es, en una ciudad que fomenta la educación y específicamente la educación para la salud entre sus ciudadanos.

Existen diversas formas de establecer comunicación y colaboración entre escuelas saludables y ciudades saludables. Una importante posibilidad la ofrecen las salidas o actividades complementarias, que en gran parte se pueden proyectar en temas como la alimentación (mercados), la seguridad (policía, bomberos) o el ejercicio físico (instalaciones deportivas). Otra forma de colaboración consiste en el tratamiento escolar de relevantes sucesos actuales. Así, por ejemplo en Galicia el hundimiento del Prestige, en 2002, dio lugar en los primeros años de este siglo, al trabajo escolar sobre múltiples cuestiones vinculadas con la salud. Y en el momento actual, la pandemia del coronavirus se ha convertido en un agente radicalmente modificador de la actividad escolar al punto de provocar el cierre de las escuelas, la promoción de sistemas alternativos de enseñanza y un especial cuidado en el proceso de vuelta a las aulas. Es muy probable que a medio y largo plazo los centros escolares deban promover con mayor intensidad que en el pasado aprendizajes de carácter preventivo en torno a estas enfermedades y que potencien estilos de vida saludables en sus alumnos.

Los proyectos interdisciplinares de las Escuelas Asociadas a la UNESCO representan un gran ejemplo de transversalidad y colaboración, que se proyectan en principio en los ámbitos de la convivencia, los derechos humanos y el medio ambiente. En ellos es posible identificar una importante presencia implícita de la educación para la salud, dado que la convivencia pacífica y el respeto al medio ambiente constituyen núcleos en torno a los que se trabajan temas como la alimentación, la contaminación de las aguas, la defensa de microrreservas de fauna y flora, campañas de información y sensibilización sobre incendios forestales y reservas hídricas, promoción de ecoturismo, etc.

Así pues, se han identificado como escenarios de educación para la salud los centros escolares y las ciudades educadoras y saludables. Y se han sugerido como principales estrategias estimuladoras del aprendizaje, la adaptación a necesidades fundamentales y la colaboración entre los diversos agentes implicados. 


\section{REFERENCIAS BIBLIOGRÁFICAS}

Asociación Internacional de Ciudades Educadoras (2004). Carta de Las Ciudades Educadoras. Recuperado de http://www.bcn.cat/edcities/esp/carta/carta_ciudades.pdf [Consultado el 12-01-2020].

Austin, R. (2009). Deja que el mundo exterior entre en el aula. Madrid: Morata.

Benejam, P. (2003). Los objetivos de las salidas. Iber. Didáctica de las ciencias sociales, geografía e historia, (36), 7-12.

FEMP (2020) Federación Española de Municipios y Provincias: RECS (Red Española de Ciudades Saludables). Recuperado de http://recs.es [Consultado el 12-01-2020].

Freinet, C. (1973). Técnicas Freinet en la Escuela Moderna. México D.F: Ed. Siglo XXI.

García Gómez, S. y Cabanillas, M. (2015). Escolares sin excursiones: ¿una consecuencia de la crisis económica? Educar, 52(1), 33-50. DOI: https://doi.org/10.5565/rev/educar.744

Kakse, V. (2019). Proyecto del Mar Báltico, de Escuelas Asociadas de la UNESCO. Recuperado de http://www.unesco.org/new/es/archives/education/networks/ global-networks/aspnet/ flagship-projects/baltic-sea-project/ [Consultado el 13/01/2020].

Ley 10/2007 de 22 de junio de la lectura, del libro y de las bibliotecas. $B O E, \mathrm{n}^{\circ} 150$, de 23 de junio.

Linguapax (2020). Qué hacemos. Recuperado de http://www.linguapax.org/es/que-hacemos [Consultado el 04/01/2020].

LOE (2006). Ley Orgánica 2/2006 de tres de mayo de Educación. BOE, nº 106, de 4 de mayo.

LOGSE (1990). Ley Orgánica 1/1990 de 3 de octubre de Ordenación General del Sistema Educativo. $B O E, \mathrm{n}^{\circ} 238$, de 4 de octubre.

LOMCE (2013) Ley orgánica para la mejora de la calidad educativa. Ley Orgánica 8/2013, 9 de diciembre. $B O E, \mathrm{n}^{\mathrm{o}} 295$, de 10 diciembre.

MEC (2007). Orden ECI 2211/2007 de 12 de julio por la que se establece el currículo y se regula la ordenación de la Educación Primaria.

MEC (2020). Proyecto de ley orgánica por la que se modifica la ley orgánica 2/2006 de 3 de mayo de educación. Recuperado de https://www.educacionyfp.gob.es/ dam/jcr:8c7d3863aaa 7-4bbd-91b2-4d05bcb80286/apl-lomloe-web2020-03-03.pdf

MECD (2017a). Planes estratégicos de convivencia escolar y hábitos saludables. Recuperado de http://www.mecd.gob.es/prensa-mecd/actualidad/2017/03/ 20170330-sectorial

MECD (2017b). II Plan de Fomento de la Lectura 2017-2020. Recuperado de https://www. mecd.gob.es/prensa-mecd/dms/mecd/prensa-mecd/actualidad/ 2017/05/20170503-planlectura/archivo1.pdf

Morillas, A. (2006). El patrimonio en manos de los jóvenes. Educar(nos), (36), 17.

OMS (1986). Carta de Ottawa para la Promoción de la Salud. Conferencia Internacional sobre la promoción de la salud. Ottawa: OMS/Salud y Bienestar social Canadá/ Asociación canadiense de salud pública.

Red Europea de Ciudades saludables de la OMS (2017). Conferencia anual de negocios y técnica: Construyendo ciudades saludables: inclusivas, seguras, resilientes y sostenibles. Pécs, Hungría. Recuperado de www.unesco.org/educativa/asp 
Rogero, J. (2010). Movimientos de renovación pedagógica y profesionalización docente. Revista Latinoamericana de Educación Inclusiva, 4(1), 141-166. Recuperado de http://www. rinace.net/rlei/numeros/vol4-num1/art7.pdf

Rosales, C. (2005). La catástrofe del "Prestige" en la enseñanza. Innovación Educativa, (15), 1119. Recuperado de http://hdl.handle.net/10347/4405

Rosales, C. (2015). Los temas transversales en el aula: convivencia, salud e igualdad. Santiago: Andavira.

Tutor, A. (2006). La ruta del esclavo. Romper el silencio. Educar (Nos), (36), 17.

UNESCO (2014). Documento 37 C/4 Estrategia a medio plazo 2014-2021. París: UNESCO. Recuperado de http://wwwUNESCO.org/new/es/quito/vacancies [Consultado el 13$01.2020]$.

UNESCO (2020a). Proyecto Mediterráneo Occidental. Recuperado de http://www.miteco.gob.es/fr/ceneam/programas-de-educacion-ambiental/ programas [Consultado el 04/01/2020].

UNESCO (2020b). Covid 19. Response. Recuperado de https://UNESCO.org 